\title{
Commentary on the Beyond WEIRD special issue: The importance of open research practices to empirical research in the evolutionary social sciences
}

\author{
Adam R. Kenny*†
}

2021-04-16

The importance of open research practices to the evolutionary social sciences has been underscored of late (e.g. Beheim 2016; Kavanagh and Kapitány 2021). The "Beyond WEIRD" special issue has exemplary demonstrations of several practices, including, but not limited to, data archiving, code sharing, and open materials. Here, I focus on these three open research practices. I reiterate their benefits for comparative cross-cultural research in the evolutionary social sciences. I also highlight some of the challenges researchers might face in their implementation, and provide pointers to possible solutions. Finally, I list elements of open research that merit greater discussion in this area of research. For brevity, the focus here is on experimental work, although open research practices apply to other empirically based cross-cultural research more broadly.

First, how common is the implementation of open research practices in the evolutionary social sciences? An indication is provided by a recent survey of the social learning literature, including cultural evolution and evolutionary psychology, conducted by Minocher et al. (2020). The authors were able to reproduce one in four results from 1955 to 2018, i.e. to re-derive the results using the same data and methods as in the original research. Low reproducibility was primarily due to unavailable, incomplete, or ambiguous data, as well as unclear analytic pipelines, an issue compounded by a lack of scripting in a statistical language such as R. However, estimated reproducibility was around $70 \%$ for 2018 . It appears that open research practices are increasingly being adopted - a shift attributable to changes in academic norms, journal requirements, and technological capabilities.

There are several advantages to open data, code, and materials (Towse et al. 2020). Innovation is promoted through the re-use of data and code. Open practices provide opportunities for collaboration; minimally, they allow researchers not part of large research programmes to integrate work. Generally, openness ensures the transparency and credibility of the scientific record. As such, these practices should be adopted, unless opting out can be justified.

Open data can facilitate cross-cultural comparisons and meta-analyses, and can help situate a primary study within the broader literature. With researchers encouraged to expand samples beyond students from "WEIRD" contexts, MTurkers, and individuals from "small-scale societies", public data will allow comparison across populations (Apicella and Barrett 2016; Milfont and Klein 2018). Townsend et al. (2020) is an illustration of the type of cross-cultural research that could draw upon open data. The authors demonstrated that sharing in a dictator game

\footnotetext{
${ }^{*}$ Calleva Research Centre for Evolution and Human Sciences, Magdalen College, Oxford, UK \& Institute of Cognitive and Evolutionary Anthropology, University of Oxford, UK; email: adam.kenny@anthro.ox.ac.uk

${ }^{\dagger}$ Commentary on the "Beyond WEIRD" special issue in Evolution and Human Behavior. This is the author accepted manuscript version of Kenny (2021) doi:10.1016/j.evolhumbehav.2021.02.008. This work is available under a CC BY-NC-ND 4.0 licence.
} 
among the Ik of Uganda was similar to behaviour in 18 other societies, the latter derived from a meta-analysis.

Open materials allow for both direct replications and checks on generalisability. A direct replication, an attempt to estimate the same effect using the same methods and population as a previous study, might be a necessary first step when a finding is novel or unexpected. Generalisability tests, possible through conceptual replications that modify methods and multisite studies, are important in building general models of human nature. A discussion of crosscultural generalisability is in Tiokhin et al. (2019), where adapted protocols for measuring social discounting were implemented with rural Bangladeshis, rural Indonesians, and undergraduates in the USA.

Direct replications might be challenging where data are collected using small and/or nonprobability samples from populations experiencing substantial social, cultural, and environmental change. For instance, the Hadza hunter-gatherers of Tanzania are considered a population in transition (Smith and Apicella 2020), with increasing exposure to outsiders and non-Hadza institutions. Yet this should not prevent studies from being replicated in theory. Furthermore, open materials can facilitate longitudinal studies and replication attempts in the (distant) future, both of which will be crucial to assess the effects of both long-term processes, like market integration and climate change, and sudden shocks, such as the COVID-19 pandemic.

Privacy and anonymity are legitimate concerns when disclosing data, particularly when information relates to either small or at-risk communities in which participants are potentially identifiable. In such cases, solutions include providing redacted data, where sensitive information has been removed, and managing access through licences that stipulate conditions of use. Alternatively, synthetic datasets can be generated: these preserve statistical properties and the original relationships between variables but there is no "real" participant. For example, Gettler et al. (2020) generated synthetic data to protect the identities of Congolese BaYaka and Bondongo men included in their study, using the R package synthpop (Quintana 2020). In doing so, results can be reproduced and effect sizes can be used in future research. If disclosing data still involves a potential risk to participants, then researchers should clearly state why. Processed data, used to complete the final analysis, should be shared, where processing is minimal. Generally, the data should be as open as possible and as closed as necessary.

Another risk to participants is that data are re-purposed for research they did not or would not consent to. Perhaps a participant would object to a secondary analyst making comparisons across groups. Relatedly, there are ethical considerations with previously collected information where consent was silent on data sharing. Neither issue prevents making data available: access and usage conditions can regulate re-use (e.g. McElreath 2020), while sharing is easier where original consent procedures did not include a promise not to share. The degree of openness should be determined on a case-by-case basis, balancing potential participant concerns with the need for a reproducible knowledge base. For a discussion and practical tips, see Meyer (2018).

Cross-cultural research can anticipate data sharing issues by engaging with communities before data collection and dissemination. How to discuss and present research practices will vary across societies and studies. Generally, discussions should be as explicit as possible with respect to usage and access, raising what to share (e.g. individual or aggregated data) and who might have access to datasets, whether other researchers, media, and/or governments. To 
derive a contextually appropriate consent procedure, community leaders and local collaborators should be included, and community members could be interviewed during pilot investigations. All the while, researchers should be attentive to data sovereignty (an issue raised in biological anthropology that applies more broadly, see Tsosie et al. 2020).

The open practices discussed here are perhaps easiest to implement with experimental research. Observational and ethnographic studies generate data that are seemingly harder to share. However, digitisation and archiving improves reproducibility and the long-term utility of such data, as highlighted by Kraft et al. (2020) in relation to decades-long research with Batek hunter-gatherers in Malaysia. Code that clearly documents the data wrangling process helps reproduce cross-cultural research that uses secondary sources.

Finally, to foster an inclusive, collaborative, and more credible evolutionary social science, the following aspects of open research practices merit further consideration:

Community involvement Researchers should discuss data sharing with study communities early in the research programme (Broesch et al. 2020). Ownership, data format, and level of anonymisation will differ depending on community needs and demands. The principle "share data" applies to both academic networks and the communities researchers work with. With shared access to data, asking and addressing questions is more collaborative.

Software licences A licence details permitted uses of the code and how others might redistribute it (Fortunato and Galassi 2021). Without an appropriate licence, it might be that researchers cannot technically use the code. A licence should be included with distributed code or software.

Training Several standards, tools, and resources facilitate open research practices (some are listed in Towse et al. 2020; Turner et al. 2020). Training should be central to educational programmes in the evolutionary social sciences, in order to develop general skills, such as writing clear metadata (e.g. description of variables), documenting code, and specifying the computational environment in which analyses were conducted, and specific computational skills for maintaining data provenance and minimising copy-paste errors, like using version control and dynamic documents.

Credit Many evolutionary social scientists undertake substantial effort to document behaviour during fieldwork. Using available data is relatively cost-free. Thus, researchers should be rewarded for sharing data (Pierce et al. 2019), through appropriate credit (e.g. the data curation role as part of the Contributor Roles Taxonomy) and citation of persistent identifiers (e.g. a digital object identifier). Data management roles should be valued and remunerated.

To paraphrase a characterisation of food sharing (Jaeggi and Gurven 2013), data sharing can be defined as the unresisted transfer of data from one data-motivated individual to another. Empirical researchers are motivated by data and rarely should there be a need to request data, code, and materials. Open research practices should be a staple of evolutionary studies of the human experience. 


\section{Acknowledgements}

I thank Rhea Arini, Malika Ihle, Laura Fortunato, Chris Osborne, Ilse Pit, Sarah de Vries, and the Editors for helpful comments, and ReproducibiliTea Oxford for discussions over the years.

\section{References}

Apicella, C. L., and H. C. Barrett. 2016. "Cross-cultural evolutionary psychology". Evolutionary Psychology, Current Opinion in Psychology 7:92-97. doi:10.1016/j.copsyc.2015.08.015.

Beheim, B. A. 2016. "Reproducible research as our new default". Anthropology News 57 (5): e57-e58. doi:10.1111/j.1556-3502.2016.570523.x.

Broesch, T., A. N. Crittenden, B. A. Beheim, A. D. Blackwell, J. A. Bunce, H. Colleran, K. Hagel, M. Kline, R. McElreath, R. G. Nelson, A. C. Pisor, S. Prall, I. Pretelli, B. Purzycki, E. A. Quinn, C. Ross, B. Scelza, K. Starkweather, J. Stieglitz, and M. Borgerhoff Mulder. 2020. "Navigating cross-cultural research: methodological and ethical considerations". Proceedings of the Royal Society B: Biological Sciences 287 (1935): 20201245. doi:10.1098/rspb. 2020. 1245 .

Fortunato, L., and M. Galassi. 2021. "The case for free and open source software in research and scholarship". Philosophical Transactions of the Royal Society A: Mathematical, Physical and Engineering Sciences 379 (2197): 20200079. doi:10.1098/rsta.2020.0079.

Gettler, L. T., S. Lew-Levy, M. S. Sarma, V. Miegakanda, and A. H. Boyette. 2020. "Sharing and caring: testosterone, fathering, and generosity among BaYaka foragers of the Congo Basin". Scientific Reports 10 (1): 1-14. doi:10.1038/s41598-020-70958-3.

Jaeggi, A. V., and M. Gurven. 2013. "Natural cooperators: food sharing in humans and other primates". Evolutionary Anthropology: Issues, News, and Reviews 22 (4): 186-195. doi:10. 1002/evan.21364.

Kavanagh, C., and R. Kapitány. 2021. "Promoting the benefits and clarifying misconceptions about preregistration, preprints, and open science for the Cognitive Science of Religion". Version 2, Journal for the Cognitive Science of Religion 6 (1-2): 130-157. doi:10 . 1558/ jcsr. 38713.

Kenny, A. R. 2021. "Commentary on the beyond WEIRD special issue: the importance of open research practices to empirical research in the evolutionary social sciences". Evolution and Human Behaviour. doi:10.1016/j. evolhumbehav.2021.02.008.

Kraft, T. S., V. V. Venkataraman, K. L. Endicott, and K. M. Endicott. 2020. "Preserving quantifiable ethnographic records of disappearing human lifeways". Evolutionary Anthropology: Issues, News, and Reviews 29 (3): 117-124. doi:10.1002/evan.21835.

McElreath, R. 2020. Data sharing, caring and access conventions. https://gist.github.com/ rmcelreath/557c5dcc7fb3ec6264088b8191772ed3. Revision 4.

Meyer, M. N. 2018. "Practical tips for ethical data sharing". Advances in Methods and Practices in Psychological Science 1 (1): 131-144. doi:10.1177/2515245917747656. 
Milfont, T. L., and R. A. Klein. 2018. "Replication and reproducibility in cross-cultural psychology". Journal of Cross-Cultural Psychology 49 (5): 735-750. doi:10.1177/0022022117744892.

Minocher, R., S. Atmaca, C. Bavero, R. McElreath, and B. A. Beheim. 2020. Reproducibility improves exponentially over 63 years of social learning research. Version 3. doi:10.31234/ osf.io/4nzc7.

Pierce, H. H., A. Dev, E. Statham, and B. E. Bierer. 2019. "Credit data generators for data reuse". Nature 570 (7759): 30-32. doi:10.1038/d41586-019-01715-4.

Quintana, D. S. 2020. "A synthetic dataset primer for the biobehavioural sciences to promote reproducibility and hypothesis generation". eLife 9:e53275. doi:10.7554/eLife.53275.

Smith, K. M., and C. L. Apicella. 2020. "Partner choice in human evolution: the role of cooperation, foraging ability, and culture in Hadza campmate preferences". Evolution and Human Behavior 41 (5): 354-366. doi:10.1016/j.evolhumbehav.2020.07.009.

Tiokhin, L., J. Hackman, S. Munira, K. Jesmin, and D. Hruschka. 2019. "Generalizability is not optional: insights from a cross-cultural study of social discounting". Royal Society Open Science 6 (2): 181386. doi:10.1098/rsos.181386.

Townsend, C., A. Aktipis, D. Balliet, and L. Cronk. 2020. "Generosity among the Ik of Uganda". Evolutionary Human Sciences 2:e23. doi:10.1017/ehs.2020.22.

Towse, J., S. Rumsey, N. Owen, P. Langford, M. Jaquiery, and C. Bolibaugh. 2020. Data sharing: a primer from UKRN. Version 1. doi:10.31219/osf.io/wp4zu.

Tsosie, K. S., J. M. Yracheta, J. Kolopenuk, and R. W. A. Smith. 2020. "Indigenous data sovereignties and data sharing in biological anthropology". American Journal of Physical Anthropology 174 (2): 183-186. doi:10.1002/ajpa.24184.

Turner, A., M. Topor, A. J. Stewart, N. Owen, A. R. Kenny, A. Jones, and D. Ellis. 2020. Open code/software: a primer from UKRN. Version 1. doi:10.31219/osf.io/qw9ck. 